\title{
Long-term results in pancreatic transplantation with special emphasis on the use of prolamine
}

\author{
W.-D. Illner ${ }^{1}$, D. Abendroth ${ }^{1}$, J. Nusser ${ }^{2}$, R. Landgraf ${ }^{2}$ and W. Land ${ }^{1}$ \\ ${ }^{1}$ Division of Transplant Surgery, Klinikum Grosshadern and ${ }^{2}$ Medical Clinic "Innenstadt", University of Munich, Munich, FRG
}

Summary Our pancreatic transplantation programme was initiated in 1979. Since then a total of 102 pancreas transplantations have been performed, blocking exocrine secretion using the duct occlusion technique with prolamine. Early non-immunological complications are frequent. The long-term results ( 9 years) in combined pancreas and kidney transplanted patients are satisfying: the survival rate for pancreas is $38 \%$ and $54 \%$ for kidney. Patient survival rate in this period is $85 \%$. Beyond the first year post-transplant the exocrine activity disappears whereas the endocrine function remains well preserved.

Key words: Pancreatic transplantation Duct-occlusion technique

\section{Introduction}

The first transplantation of the pancreas at our institution was performed in 1979 (Land 1980). It was decided to use a segmental pancreatic graft and the duct occlusion technique with an alcoholic amino acid solution (prolamine) to eliminate exocrine secretion. The pros and cons of different surgical techniques for pancreatic duct managements habe been discussed previously (Najarian 1988).

In this study we report on our long-term results of duct occluded pancreatic transplantations in simultaneously (pancreas-kidney) transplanted patients.

\section{Subjects and Methods}

Since 1979 a total of 102 pancreas transplantations using prolamine duct occlusion have been performed at our centre. In 90 patients a simultaneous pancreas and kidney transplantation was performed. Five patients received a pancreas regraft and seven patients only a pancreas. In all cases we used a segmental pancreas for grafting.
Recipient Operation. In our second series - from 1984 to 1988 - the essential surgical technique was modified as follows: strictly intraperitoneal placement of the pancreas along the ascending colon plus a continuous intra-abdominal lavage during the first and second day post-operatively The rationale behind this procedure was to minimize local complications which could be induced by the damage of prolamine occlusion like pancreatitis, oedema and transient residual exocrine secretion (Illner 1987). In addition in this series we improved the immunosuppressive protocol as well as anticoagulation therapy by introducing quadruple drug induction therapy as well as the use of Dextran 40 plus "low dose" heparin (Illner 1987; Abendroth 1989).

Characteristics of Prolamine. The mode of action of prolamine has been investigated in animal experiments by Gebhardt and Stolte (Gebhardt 1978). Prolamine destroys the exocrine pancreatic parenchyma completely while the islets of Langerhans remain unchanged and well preserved, also in man (Landgraf 1986). So far there is no reason to suspect a deleterious influence of this substance on the long-term endocrine function (Landgraf 1990).

The exocrine activity disappears at least beyond the first year posttransplant. In contrast to experimental findings we have noted a delayed destruction of exocrine parenchyma in our clinical studies (Tliner 1988). Residual exocrine secretion (minimum: 2 months, maximum: 18 months) is associated with a high rate of fistulae, especially in the early post-operative phase. A higher rate of irreversible venous thrombosis induced by prolamine is however, questionable.

\section{Results}

The overall long-term results of simultaneous pancreas and kidney transplantation are shown in Figure 1. The 9year graft survival rate is $38 \%$ for pancreas and $54 \%$ for kidney transplantation. Patient survival rate for this period is $85 \%$.

The pancreas survival rate improved using modified immunosuppressive therapy, surgical technique and anticoagulation therapy and is shown in Figure 2 . The pancreatic function rate during the period $1981-1984$ is $20 \%$ ( 5 years post-transplantation) in contrast to $40 \%$ during the period from 1984 - 1988. 
The main non-immunological complications are pancreatic fistulae (32\%), pancreatic pseudocysts (5\%) and primary irreversible venous thromboses (13\%).

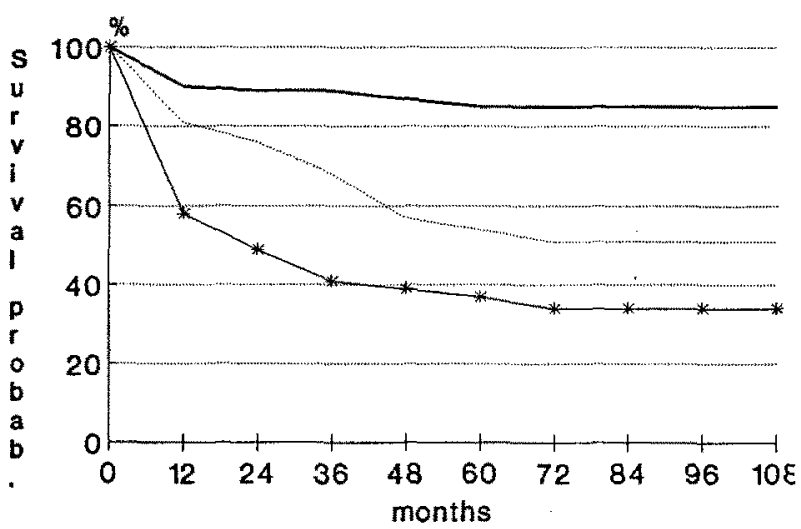

Fig. 1: Patient, pancreas and kidney survival rates in simultaneous pancreas and kidney transplantation (estimated by computerized Cutler/ Ederer-formula. Cutler and Ederer, 1958)

(patient $=\ldots$; pancreas $=\ldots$; kidney $=-*_{-}$).

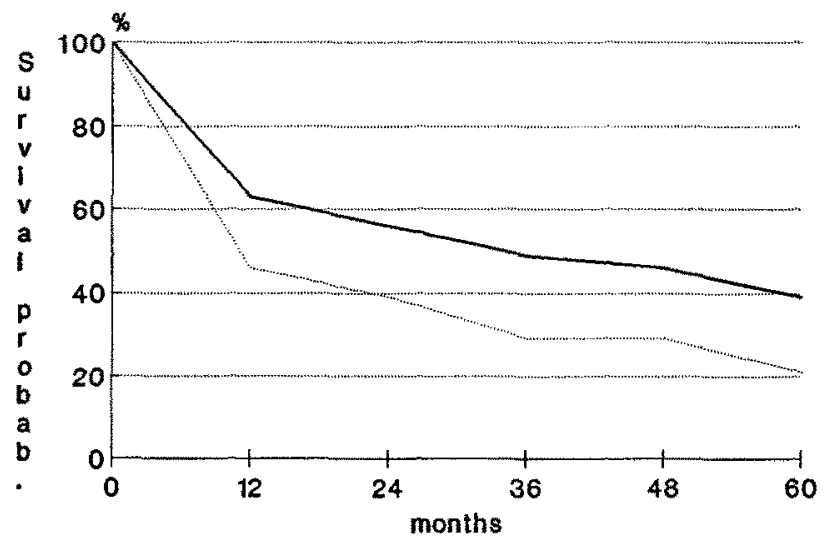

Fig. 2: Pancreas survival rate in combined pancreas and kidney transplantation, using different post-operative management (Cutler and Ederer 1958) $($ period $I=\ldots$; period $I I=\cdots)$.

\section{Discussion}

Pancreas transplantation using duct occlusion is technically simple, effective and reasonably safe for the patients. The success rate could be improved with some modifications. The long-term results of the combined transplanted group are satisfying. In comparison to the International Pancreas Transplant Registry, the 1-year pancreas survival rate is slightly higher in the bladder drained group than in others (intestinal drainage, duct occlusion (Sutherland 1991). The non- immunological rate of complication is frequent and leads to loss of pancreatic graft function in a relatively high percentage, especially in the early postoperative period. Beyond the first year of pancreatic grafting the exocrine activity disappears and the patient is free of surgical, infectious and metabolic complications.

No deterioration of endocrine function occurred during the long-term follow-up of successfully transplanted patients.

\section{References}

Abendroth D, Ilner WD, Landgraf R, Land W (1989) Modification of duct-occlusion technique in segmental pancreas transplantation. Diabetes 38 [Suppl.1] 234

Cutler SJ, Ederer F (1958) Maximum utilization of life table-method in analyzing survival. J.Chron.Dis. Vol.no.8 699-713

Gebhardt Ch, Stolte M (1978) Die Ausschaltung des exkretorischen Pankreasparenchyms đurch intraductale Injektion einer schnell härtenden Aminosäurelösung. Der Chirurg 49 [Suppl.1]: 428-430

Illner WD, Abendroth D, Landgraf R, Gokel M, Land W (1988) Experience with prolamine for duct obliteration. In: van Schilfgaarde, M.A.Hardy (eds) Transplantation of the endocrine pancreas in Diabetes mellitus. Elsevier Science, Amsterdam: 179-184

Illner WD, Abendroth D, Landgraf R, Land W (1987) Modifizierte Operationstechnik und postoperatives Management nach simultaner Pankreas- und Nierentransplantation. Langenbecks Archiv Chir. 372 [Suppl.1]: 984

Illner WD, Gottwald T, Abendroth D and Land W (1987) Incidence of fistulas following human pancreas transplantation - Positive influence of reabsorption of pancreatic secretions by the peritoneum. Transpl. Proc. 19: 2323-2324

Land W, Eberhard K, Weitz H, Gokel JM (1980) Gangokklusion mit Ethibloc bei segmentaler Pankreastransplantation. In: Gebhardt $\mathrm{Ch}$, Stolte M (eds) Pankreasgangokklusion, Verlag Gerhard Witzstroch $103-110$

Landgraf R, Landgraf-Leurs MMC, Burg W et al (1986) Long-term follow-up of segmental pancreas transplantation in Type I diabetes. Transpl.Proc. 18: 1118-1124

Landgraf R, Nusser J, Landgraf-Leurs MMC, Loy A et al (1990) Metabolic studies of Type I diabetics after successfull segmental pancreas and kidney transplantation. Transpl.Proc. 22: 1588-1590

Najarian JS (1988) Landmarks in clinical pancreatic transplantation. In: Groth CG (ed) Pancreatic Transplantation. WB Saunders Philadelphia 15-19

Sutherland DER, Gillingham K, and Moudry-Munns KC (1991) Registry report on clinical pancreas transplantation. Transp.Proc. 23: $55-57$

Dr. W.-D. Illner

Division of Transplant Surgery

Klinikum Grosshadern, University of Munich

Marchioninistr.15

8000 München 70/FRG 